\title{
Insights into kisspeptin- and leptin-signalling on GnRH mRNA expression in hypothalamic organ cultures of immature pikeperch Sander lucioperca
}

\author{
F. J. Schaefer $\cdot$ S. Wuertz
}

Received: 19 February 2016/Accepted: 13 May 2016/Published online: 25 May 2016

(C) The Author(s) 2016. This article is published with open access at Springerlink.com

\begin{abstract}
Two types of gonadotropin-releasing hormones $(\mathrm{GnRH})$ were identified as gnrh1 and gnrh2 in pikeperch Sander lucioperca. The administration of rodent leptin on hypothalamic organ cultures of immature pikeperch resulted in significantly elevated levels of gnrh2, but not in gnrh1 mRNA, whereas kisspeptin-10 administration did not affect gnrh1 or gnrh2 expression. These results represent preliminary insights into leptin-GnRH-signaling on a hypothalamic level in fish, potentially coupling fat metabolism and the activation of the reproductive axis during puberty. Mammalian leptin and kisspeptin-10, however, failed to initiate a consistent response in pikeperch and their use cannot be recommended.
\end{abstract}

Keywords Hypothalamus-pituitary-gonad axis $\cdot$ Percidae $\cdot$ Puberty $\cdot$ Reproduction

\section{Introduction}

Pikeperch, Sander lucioperca (L. 1758), is an emerging species in recirculating freshwater aquaculture and has the potential to play a major role in this sector in Europe. However, production of pikeperch in aquaculture is impeded by a shortage of fingerlings due to reproductive dysfunctions, predominantly owing to the stagnation of puberty, asynchronous maturation and low gamete quality. Consequently, reproductive management is a major focus of current research efforts. Authors recently revealed that a thermal induction of puberty is followed by a photothermal synchronization of gonad maturation (Hermelink et al. 2011, 2013). Thus, it is relatively well documented that temperature and photoperiod are the main abiotic triggers acting on the reproductive axis, involving the conserved endocrine signaling cascade from the hypothalamus via the pituitary to the gonads (HPG axis).

However, little is known about the neuroendocrine control in the brain, which ultimately activates reproduction in pikeperch. As in other vertebrates, kisspeptins have been found to play a key role in the signal transduction, which targets the expression of gonadotropin-releasing hormone $(\mathrm{GnRH})$ in the hypothalamus in fish (Tena-Sempere et al. 2012). Here, kisspeptins act as mediators at the interface of exogenous parameters and the induction of puberty and maturation (Castellano et al. 2005; Biran et al. 2008; Filby et al. 2008; Oakley et al.

F. J. Schaefer · S. Wuertz

Department of Ecophysiology and Aquaculture, Leibniz-Institute of Freshwater Ecology and Inland Fisheries,

Müggelseedamm 310, 12587 Berlin, Germany

F. J. Schaefer $(\square) \cdot$ S. Wuertz

Faculty of Life Sciences, Thaer-Institute of Agricultural and Horticultural Sciences, Humboldt-Universität zu Berlin, Invalidenstraße 42, 10115 Berlin, Germany

e-mail: schaefer@igb-berlin.de 
2009; Zohar et al. 2010). For example, in juvenile chub mackerel, Scomber japonicus Houttuyn 1782, which express two forms of the kiss gene (kiss1 and kiss2), kiss1 in males and kiss1 and kiss2 in females increased together with gnrh1 levels at the initiation of puberty, at the onset of meiosis in the testes and the onset of vitellogenesis in females, respectively (Ohga et al. 2015). In higher vertebrates, kisspeptins transduce endogenous signals on the nutritional status of the organism via leptin towards the HPG axis (Oakley et al. 2009), since kisspeptin-expressing neurons were identified as direct targets of leptin regulating GnRH expression (Smith et al. 2006). Furthermore, leptin-specific receptors (lepr) have been identified in the hypothalamus of zebrafish, Danio rerio (Hamilton 1822) (Liu et al. 2010). Yet, several studies indicate that the dependence of maturation on the nutritional status and mobilization of fat reserves is preceding the initiation of puberty (Rowe et al. 1991; Shearer and Swanson 2000; Silverstein et al. 1997; Thorpe 1994). However, a direct link between the activation of the HPG axis and leptin is yet to be reported in fish. Recombinant human leptin induced follicle stimulating hormone (FSH) and luteinizing hormone (LH) release in rainbow trout, Onchorhynchus mykiss (Walbaum 1792), in vitro, but only in post-pubertal pituitaries (Weil et al. 2003).

Since it is commonly assumed that the activation of the pituitary is initiated through hypothalamic GnRH release and an involvement of leptin on such GnRH release via kisspeptins was observed in mammals, it is most likely to detect a similar pattern in fish (Copeland et al. 2011; Taranger et al. 2010). To date, hatchery protocols for pikeperch and other teleost fish often include hormone treatments (e.g., human chorionic gonadotropin, carp pituitary extract, GnRH analogs) for the induction of puberty, gonad maturation or for the synchronization of spawning (Żarski et al. 2015). Still, reproduction is a key problem for the development of the industry, particularly for year-round production in recirculating aquaculture (Wuertz et al. 2012). Therefore, the endocrine inter-linkage of nutrition, fat metabolism and reproduction represented by the leptinkiss-GnRH pathways can be useful with regards to improved hormone treatments and more importantly, as tool to optimize broodstock nutrition.

This study tests the effect of rodent leptin and kisspeptin-10 (YNWNSFGLRY-NH ${ }_{2}$ ) on GnRH expression in hypothalamic organ cultures of immature pikeperch. It seeks to address direct effects on the hypothalamus in vitro in pre-pubertal brains (absence of thermal stimulation).

\section{Materials and methods}

Sampling and organ cultures

A group of 15 1-year old immature pikeperch (three male, nine female, three undifferentiated) with an average weight of $267.5 \mathrm{~g}$ were killed by cutting the spine. The brain of each fish was dissected and the hypothalamus was gently removed. To facilitate diffusion in organ culture, the hypothalamus was cut sagittal in half and each half was transferred into $1 \mathrm{ml}$ of cooled culture medium.

Hypothalamic organ cultures were performed according to a technique reported by Leal et al. (2009) for zebrafish. In brief, $750 \mu \mathrm{l}$ agarose cylinders (1.5\% agarose in $\mathrm{pH} 7.4$ Ringers solution) were formed in 48-well-flat bottom plates, autoclaved, pre-soaked overnight in culture medium and covered with a $0.25 \mathrm{~cm}^{2}$ nitrocellulose membrane $(25 \mu \mathrm{m}$ thickness, $0.22 \mu \mathrm{m}$ pore size; Millipore) inside 24-well-flat bottom plates. DMEM culture medium was prepared according to Opitz et al. (2006). All procedures were performed under sterile conditions.

Prior to the incubation of the organs, wells containing agarose cylinders were filled with $1.5 \mathrm{ml}$ of culture medium. Control wells were filled with culture medium only (six replicates), while treatments contained 1, 10 or $100 \mathrm{nmol} \mathrm{l}^{-1}$ of rodent leptin (Shenandoah Biotechnology) or kisspeptin-10 (AnaSpec) with four replicates per treatment. Hypothalamus-halves were randomly distributed (in total 30 wells) and the cultures were incubated for $24 \mathrm{~h}$ in a cell culture cabinet (Binder) at $20{ }^{\circ} \mathrm{C}$. After incubation, organs were immediately transferred into $1 \mathrm{ml}$ RNAlater reagent (Qiagen) and stored at $-80{ }^{\circ} \mathrm{C}$ after overnight incubation at $4{ }^{\circ} \mathrm{C}$.

qPCR analysis

The RNA extraction with TRIzol (Thermo Fisher Scientific) and chloroform was performed according to Kroupova et al. (2011). Tissue was homogenized with a TissueLyser (Qiagen). The RNA concentration was measured by UV absorption spectrometry using a Nanodrop ND-1000 spectrophometer (Thermo Fisher 
Table 1 Conserved primers (cons) and specifications for the RT-QPCR assays for gnrh1 and gnrh2, using rpl8 as a housekeeping gene, forward (f) and reverse (r) primers, primer-specific product length in base pairs (bp), GenBank accession number (GenBank \#), annealing temperature (TA) and PCR efficiency (Eff)

\begin{tabular}{lllllll}
\hline Target gene & Primer & $5^{\prime}-3^{\prime}$ sequence & TA $\left({ }^{\circ} \mathrm{C}\right)$ & Size $(\mathrm{bp})$ & GenBank \# & Eff $(\%)$ \\
\hline cons & $\mathrm{f}$ & GCACTGGTCSTATGGACTGAGT & & & & \\
gnrh1 & $\mathrm{r}$ & TCATTTYTTGTAHGTTCTGBGTCCG & & & & \\
cons & $\mathrm{f}$ & TGCTYGGGCTGCTTCTATGT & & & & \\
gnrh2 & $\mathrm{r}$ & CYCCTCCTCTGGGGTCTCA & & & & \\
gnrh1 & $\mathrm{f}$ & TCTGGACAGCCTTTCAGAC & 53 & 170 & KC692519 & 92.4 \\
& $\mathrm{r}$ & GACGAAGGGTGATTCCTCTAC & & & & \\
gnrh2 & $\mathrm{f}$ & GCTCAGCTGTCCAATGCC & 58 & 136 & KC692520 & 94.9 \\
& $\mathrm{r}$ & GCCTCACACAGCTTAATCTCC & & & & \\
rpl8 & $\mathrm{f}$ & GTTATCGCCTCTGCCAC & 62 & 167 & HQ259050 & 96.5 \\
& $\mathrm{r}$ & ACCGAAGGGATGCTCAAC & & & & \\
\hline
\end{tabular}

Scientific). Integrity number for of the RNA (RIN) was determined for a subset of ten samples on a RNA 6000 Nano LabChip in an Agilent 2100 Bioanalyzer (Agilent Technologies). All samples were diluted to a final

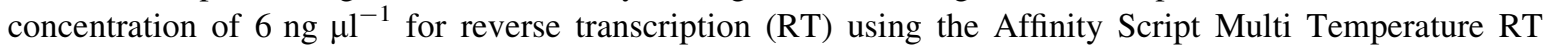
(Agilent).

First, two paralogous mRNAs of GnRH were identified from hypothalamus mRNA extracts as gnrh1 (KC692519) and gnrh2 (KC692520), using conserved primers (consgnrh1, consgnrh2) designed from sequence information available (Table 1). After direct sequencing (Seqlab, Germany), amplicons were confirmed by alignment analysis, using available sequence information of Perciformes. RT-QPCR primers were designed from the respective sequences and confirmed by direct sequencing as described. Primers for rpl8 as a housekeeping gene have been published by Hermelink et al. (2011). Quantification assays included $2 \mu 1$ cDNA, 1 U Platinum Taq polymerase (Invitrogen), $0.125 \mu \mathrm{l}$ of 100 fold diluted SYBR-Green solution (Invitrogen), $5 \mathrm{mM} \mathrm{MgCl} 2,1 \times$ buffer, $0.2 \mathrm{mmol} \mathrm{dNTP}$ and $10 \mathrm{pmol}$ of each primer (temperature protocol: 10 min degeneration at $96{ }^{\circ} \mathrm{C}$, followed by 40 cycles of $30 \mathrm{~s}$ degeneration $\left(96{ }^{\circ} \mathrm{C}\right), 20 \mathrm{~s}$ annealing $\left(62{ }^{\circ} \mathrm{C}\right.$ for rp18, $53{ }^{\circ} \mathrm{C}$ for gnrh1, $58{ }^{\circ} \mathrm{C}$ for gnrh2), $20 \mathrm{~s}$ elongation at $72{ }^{\circ} \mathrm{C}$ ). Quantification assays were performed in duplicate. Absolute quantification was carried out with a standard dilution series of the PCR product (after gel extraction) using the DNA concentration and the molecular weight of the amplicon to calculate the number of copies. Data were normalized to rpl8 as housekeeping gene.

Statistical analysis was performed with GraphPad Prism (GraphPad Software), using one-tailed, nonparametric Kruskal-Wallis and Dunn's multiple comparison tests and linear regression.

\section{Results and discussion}

Despite a single peak in one individual organ culture, caused by leptin administration at a concentrations of $1 \mathrm{nmol} 1^{-1}$, no significant differences $(p=0.25)$ in gnrh1 mRNA expression were recorded in response to both hormones, irrespective of the applied concentration (Fig. 1). In contrast, expression of gnrh2 revealed a significant increase in leptin-treated organ cultures compared to the control in all concentrations (0.023 copies/copy rpl8 at $1 \mathrm{nmol} 1^{-1}, 0.013$ at $10 \mathrm{nmol} \mathrm{l}^{-1}$ and 0.023 at $100 \mathrm{nmol} \mathrm{l}^{-1}$ leptin; $p<0.05$ ). Kisspeptin-10 did not effect gnrh1 or gnrh2 expression. Interestingly, gnrh1 and gnrh2 expression were not correlated $\left(R^{2}=0.0023\right)$.

Although the role of leptin in reproduction is relatively well studied in mammals and despite the critical importance of the nutrition-reproduction nexus within ecology and aquaculture, leptin-mediated effects on reproduction is poorly understood in fish and limited to effects on the pituitary level (Peyon et al. 2001; Weil et al. 2003). Watanobe (2002) demonstrated that intra-hypothalamic perfusions of leptin resulted in elevated GnRH secretion in rats, Rattus rattus L. 1758. This is most likely mediated via kisspeptins, since GnRH neurons do not express leptin receptors in mammals (cf. review by Elias 2012). Here, an elevation of gnrh2 is 

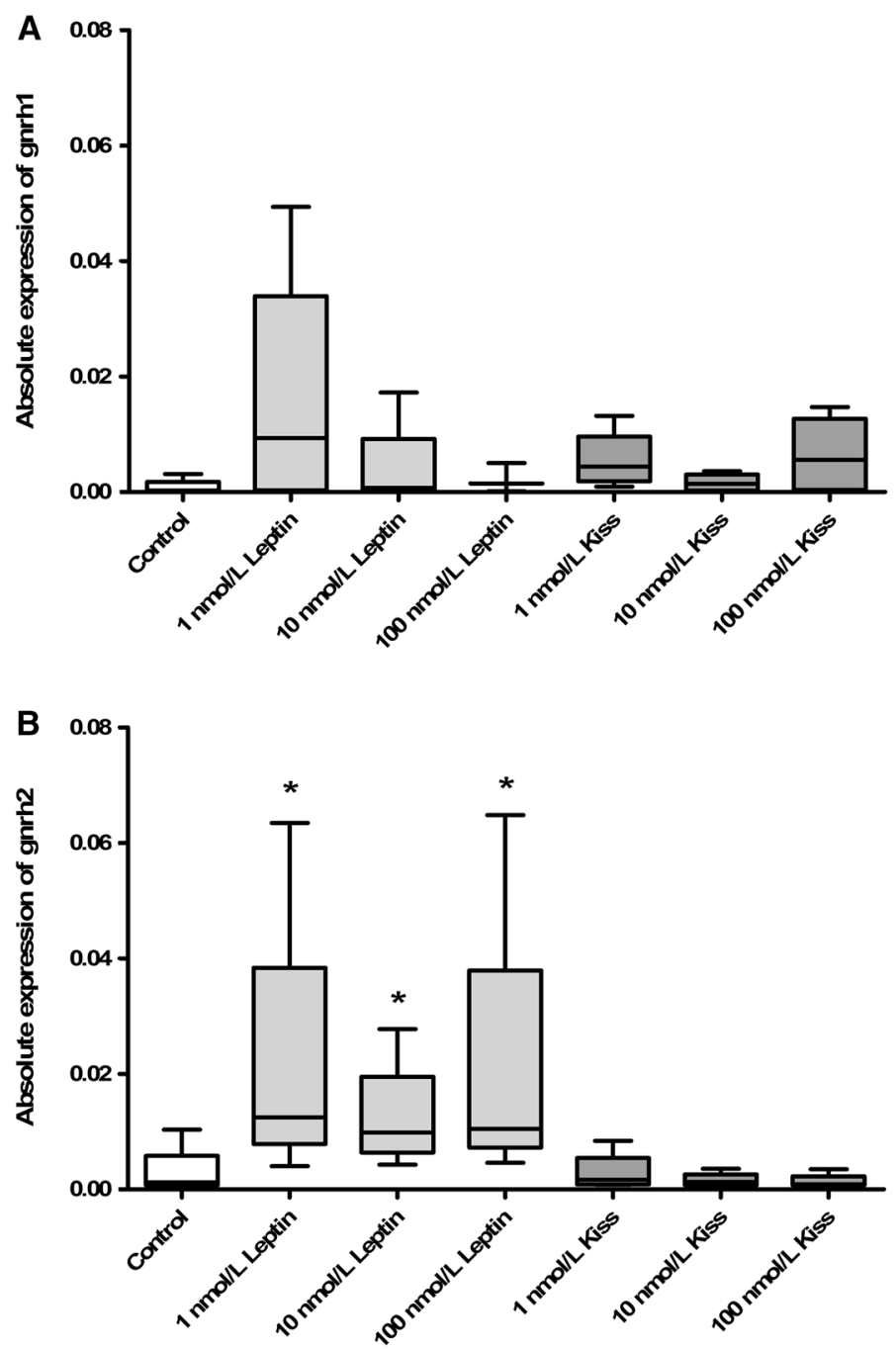

Fig. 1 Boxplot of absolute mRNA expression of gnrh1 (a) and gnrh2 (b) in the hypothalamus at 1, 10 or $100 \mathrm{nmol} 1^{-1}$ of rodent leptin or kisspeptin 10 in organ cell culture. Columns indicate 25th and 75th percentile and contain the median (line). Whiskers indicate the minimum and maximum values. Gene expression was normalized to rpl8 and presented as copy target gene per copy rpl8. Significant differences $(p<0.05)$ of treatments compared to the control are marked with an asterisk (Kruskal-Wallis, Dunn's test)

reported, which suggests a leptin mediated activation of the HPG axis similar to the widely accepted model in mammals.

Paralogous GnRH forms have been identified in several fish species and differential regulation suggests a sub-functionalization of these paralogs (Zohar et al. 2010). Here, leptin only induced up-regulation of one isoform, previously identified as gnrh2 by sequence comparison, suggesting that gnrh2 may be the prime target of leptin. Notably, gnrh1 increased in response to leptin, but did not reveal significance due to the high variability observed. With regard to the data on the sub-functionalization of $\mathrm{GnRH}$, it has been suggested that one paralog activates the HPG axis. Furthermore, we propose that gnrh2 is the key player regulating hypophyseal gonadotropes (hypophysiotrophic) and in particular inducing puberty in pikeperch. This assertion is in agreement with the mammalian model of puberty induction and is supported by the hypothesis on the lipostatic induction of puberty. In addition to a key role in reproduction, gnrh2 exerts anorexic effects in zebrafish (Nishiguchi et al. 2012) and goldfish, Carassius auratus (L. 1758) (Kang et al. 2011; Matsuda et al. 2008). Therefore, the observed up-regulation of gnrh 2 mRNA may indicate a possible involvement of gnrh 2 in leptin-mediated anorexigenic effects (Londraville et al. 2014). 
In this study, rodent kisspeptin did not confirm direct effects on either GnRH paralog. The roles of the two kisspeptin transcripts kiss1-10 and kiss2-10 are contradictory in fish, as reviewed in detail by Tena-Sempere et al. (2012) and Parhar et al. (2012). In zebrafish, injections of kiss1-10 (YNWNSFGLRY, corresponding to rodent kisspeptin used here) and kiss2-10 (FNFNPFGLRF) had no effect on gnrh3 (hypophysiotropic form in zebrafish), as well as gnrh2 in adult females (Kitahashi et al. 2009), despite inducing an increase of hypothalamic gnrh1 expression in sexually mature orange spotted grouper, Epinephelus coioides (Hamilton 1822) (Shi et al. 2010).

Rodent kisspeptin-10 (corresponding to kiss1-10) stimulated neither gnrh1, nor gnrh2 expression in the hypothalamus of pikeperch. Since the hypophysiotropic form of GnRH in pikeperch is unknown at present, it remains to be determined whether a third undetected form (e.g., gnrh3) is the hypophysiotropic target of kisspeptin-10 in this species. Alternatively, kiss2 may be the predominant regulator of gonadotropin release in fish, as suggested by Kitahashi et al. (2009). Consequently, rodent kisspeptin failed to induce GnRH transcription here.

Yet, an increase in gnrh2 in response to leptin has been observed, suggesting that gnrh2 is the hypophysiotropic form of GnRH in pikeperch. Thus, it is plausible to conclude that this effect is transduced via kisspeptins and with regard to the failure of rodent kiss1-10 used it is conceivable that the transcript of the kiss 2-10 is the effective kisspeptin activating the HPG axis by inducing GnRH synthesis. However, this assumption is a tentative one. It is necessary to conduct additional (in vivo) studies to further examine the role of kisspeptins and leptin on GnRH mRNA expression and GnRH secretion in the pituitary of pikeperch and their involvement in reproduction. However, the use of mammalian (rodent) kisspeptin or leptin cannot be recommended, hence, native recombinant hormones need to be explored in the future.

Acknowledgments This study was funded by the IGB Fellowship Program in Freshwater Science.

Open Access This article is distributed under the terms of the Creative Commons Attribution 4.0 International License (http:// creativecommons.org/licenses/by/4.0/), which permits unrestricted use, distribution, and reproduction in any medium, provided you give appropriate credit to the original author(s) and the source, provide a link to the Creative Commons license, and indicate if changes were made.

\section{References}

Biran J, Ben-Dor S, Levavi-Sivan B (2008) Molecular identification and functional characterization of the kisspeptin/kisspeptin receptor system in lower vertebrates. Biol Reprod 79:776-786

Castellano J, Navarro V et al (2005) Changes in hypothalamic KiSS-1 system and restoration of pubertal activation of the reproductive axis by kisspeptin in undernutrition. Endocrinol 146:3917-3925

Copeland DL, Duff RJ, Liu Q, Prokop J, Londraville RL (2011) Leptin in teleost fishes: an argument for comparative study. Front Physiol 2:26

Elias CF (2012) Leptin action in pubertal development: recent advances and unanswered questions. Trends Endocrinol Metab 23:9-15

Filby AL, van Aerle R, Duitman J, Tyler CR (2008) The kisspeptin/gonadotropin-releasing hormone pathway and molecular signaling of puberty in fish. Biol Reprod 78:278-289

Hermelink B, Wuertz S, Trubiroha A, Rennert B, Kloas W, Schulz C (2011) Influence of temperature on puberty and maturation of pikeperch, Sander lucioperca. Gen Comp Endocrinol 172:282-292

Hermelink B, Wuertz S, Rennert B, Kloas W, Schulz C (2013) Temperature control of pikeperch (Sander lucioperca) maturation in recirculating aquaculture systems-induction of puberty and course of gametogenesis. Aquaculture 400:36-45

Kang KS, Shimizu K et al (2011) Gonadotropin-releasing hormone II (GnRH II) mediates the anorexigenic actions of $\alpha$ melanocyte-stimulating hormone $(\alpha-\mathrm{MSH})$ and corticotropin-releasing hormone $(\mathrm{CRH})$ in goldfish. Peptides 32:31-35

Kitahashi T, Ogawa S, Parhar IS (2009) Cloning and expression of kiss2 in the zebrafish and medaka. Endocrinol 150:821-831

Kroupova H, Trubiroha A, Wuertz S, Kloas W (2011) Stage-dependent differences in RNA composition and content affect the outcome of expression profiling in roach (Rutilus rutilus) ovary. Comp Biochem Physiol 159:141-149

Leal MC, de Waal PP, Garcia-Lopez A, Chen SX, Bogerd J, Schulz RW (2009) Zebrafish primary testis tissue culture: an approach to study testis function ex vivo. Gen Comp Endocrinol 162:134-138

Liu Q, Chen Y, Copeland D, Ball H, Duff RJ, Rockich B, Londraville RL (2010) Expression of leptin receptor gene in developing and adult zebrafish. Gen Comp Endocrinol 166:346-355

Londraville RL, Macotela Y, Duff RJ, Easterling MR, Liu Q, Crespi EJ (2014) Comparative endocrinology of leptin: assessing function in a phylogenetic context. Gen Comp Endocrinol 203:146-157

Matsuda K, Nakamura K et al (2008) Inhibitory effect of chicken gonadotropin-releasing hormone II on food intake in the goldfish, Carassius auratus. Horm Behav 54:83-89 
Nishiguchi R, Azuma M, Yokobori E, Uchiyama M, Matsuda K (2012) Gonadotropin-releasing hormone 2 suppresses food intake in the zebrafish, Danio rerio. Front Endocrinol 3:122

Oakley AE, Clifton DK, Steiner RA (2009) Kisspeptin signaling in the brain. Endocr Rev 30:713-743

Ohga H, Adachi H et al (2015) mRNA levels of kisspeptins, kisspeptin receptors, and GnRH1 in the brain of chub mackerel during puberty. Comp Biochem Physiol 179:104-112

Opitz R, Trubiroha A et al (2006) Expression of sodium-iodide symporter mRNA in the thyroid gland of Xenopus laevis tadpoles: developmental expression, effects of antithyroidal compounds, and regulation by TSH. J Endocrinol 190:157-170

Parhar I, Ogawa S, Kitahashi T (2012) RFamide peptides as mediators in environmental control of GnRH neurons. Prog Neurobiol 98:176-196

Peyon P, Zanuy S, Carrillo M (2001) Action of leptin on in vitro luteinizing hormone release in the European sea bass (Dicentrarchus labrax). Biol Reprod 65:1573-1578

Rowe DK, Thorpe JE, Shanks AM (1991) Role of fat stores in the maturation of male Atlantic salmon (Salmo salar) parr. Can J Fish Aquat Sci 48:405-413

Shearer KD, Swanson P (2000) The effect of whole body lipid on early sexual maturation of $1+$ age male chinook salmon (Oncorhynchus tshawytscha). Aquacult 190:343-367

Shi Y, Zhang Y et al (2010) Molecular identification of the kiss2/kiss1ra system and its potential function during 17alphamethyltestosterone-induced sex reversal in the orangespotted grouper, Epinephelus coioides. Biol Reprod 83:63-74

Silverstein JT, Shimma H, Ogata H (1997) Early maturity in amago salmon (Oncorhynchus masu ishikawai): an association with energy storage. Can J Fish Aquat Sci 54:444-451

Smith JT, Acohido BV, Clifton DK, Steiner RA (2006) KiSS-1 neurones are direct targets for leptin in the ob/ob mouse. J Neuroendocrinol 18:298-303

Taranger GL, Carrillo M et al (2010) Control of puberty in farmed fish. Gen Comp Endocrinol 165:483-515

Tena-Sempere M, Felip A, Gomez A, Zanuy S, Carrillo M (2012) Comparative insights of the kisspeptin/kisspeptin receptor system: lessons from non-mammalian vertebrates. Gen Comp Endocrinol 175:234-243

Thorpe JE (1994) Reproductive strategies in Atlantic salmon, Salmo salar L. Aquac Fish Manage 25:77-87

Watanobe H (2002) Leptin directly acts within the hypothalamus to stimulate gonadotropin-releasing hormone secretion in vivo in rats. J Physiol 545:255-268

Weil C, Le Bail PY, Sabin N, Le Gac F (2003) In vitro action of leptin on FSH and LH production in rainbow trout (Onchorynchus mykiss) at different stages of the sexual cycle. Gen Comp Endocrinol 130:2-12

Wuertz S, Hermelink B, Schulz C (2012) Pike perch in recirculation aquaculture. Glob Aquac Alliance 15:20-21

Żarski D, Horváth Á, Held JA, Kucharczyk D (2015) Artificial Reproduction of Percid Fishes. In: Kestemont P, Dabrowski K, Summerfelt RC (eds) Biology and culture of percid fishes. Springer Verlag, Dordrecht, pp 123-162

Zohar Y, Muñoz-Cueto JA, Elizur A, Kah O (2010) Neuroendocrinology of reproduction in teleost fish. Gen Comp Endocrinol 165:438-455 